\title{
ARTí́CULO
}

\section{Aves marinas varadas en la bahía de Coquimbo, norte de Chile: ¿Qué especies y cuántas mueren?}

Beached seabirds in Coquimbo Bay, northern Chile: What species and how many die?

\author{
Matías Portflitt-Toro ${ }^{1,2}$, Diego Miranda-Urbina ${ }^{3}$ y Guillermo Luna-Jorquera ${ }^{2,4, *}$
}

\author{
'Programa de Magíster en Ciencias del Mar mención Recursos Costeros, Facultad de Ciencias del Mar, Universidad Católica \\ del Norte, Larrondo 1281, Coquimbo, Chile \\ ${ }^{2}$ Millennium Nucleus for Ecology and Sustainable Management of Oceanic Islands (ESMOI), Departamento de Biología \\ Marina, Universidad Católica del Norte, Larrondo 1281, Coquimbo, Chile \\ ${ }^{3}$ Instituto de Ciencias Biológicas, Universidad de Talca, Av. Lircay S/N, Talca, Chile \\ ${ }^{4}$ Centro de Estudios Avanzados en Zonas Áridas (CEAZA), Coquimbo, Chile. *Autor corresponsal: gluna@ucn.cl
}

\begin{abstract}
Monitoring of seabirds stranded on beaches can provide information about their causes of death, such as bycatch in fishing activities or oil spills. During a year we monthly monitored the Coquimbo Bay, north Chile, to quantify the number of seabirds and species stranded. We found 395 dead seabirds, of which 382 were identified and grouped into 21 species. The most abundant were the Peruvian booby with 115 individuals (30\%), the Guanay cormorant with 83 individuals (22\%) and the Kelp gull with 65 individuals (17\%). The most abundant and frequent species were those nesting in the Coastal System of Coquimbo. Mortality mainly affected seabirds that feed on anchoveta, which is the main target resource of purse seine fishing. We suggest that the incidental mortality caused by fishing is chronic and relatively constant during the year. A long-term monitoring program is needed to better estimate the mortality and the factors that influence the interaction of seabirds with fishing gears.
\end{abstract}

Key words: Strandings, seabirds, Suliformes, Coastal System of Coquimbo, Chile

Resumen.- El monitoreo de las aves varadas en las playas puede proporcionar información sobre sus causas de muerte, tales como la captura incidental en actividades de pesca o derrames de petróleo. Durante un año fue monitoreada mensualmente la Bahía de Coquimbo, norte de Chile para cuantificar el número de aves marinas varadas. Se encontraron 395 aves marinas muertas de las cuales 382 fueron identificadas y agrupadas en 21 especies. Las especies más abundantes fueron los piqueros con 115 individuos (30\%), el cormorán guanay con 83 individuos (22\%) y la gaviota dominicana con 65 individuos (17\%). Las especies más abundantes y frecuentes fueron las que anidan en el Sistema Costero de Coquimbo. La mortalidad afecta principalmente a las aves marinas que se alimentan de la anchoveta, que es el principal recurso objetivo de la pesca con redes de cerco. Se sugiere que la mortalidad incidental causada por la pesca es crónica y relativamente constante durante el año. Se requiere un programa de monitoreo a largo plazo para mejorar la estimación de la mortalidad y los factores que influyen en la interacción de las aves marinas con las artes de pesca.

Palabras clave: Varamientos, aves marinas, Suliformes, Sistema Costero de Coquimbo, Chile

\section{INTRODUCCIÓN}

Las poblaciones de aves marinas comparadas con otros grupos de aves en general están disminuyendo a una alta tasa (Croxall et al. 2012), por lo que es fundamental determinar las amenazas que provocan la reducción de sus poblaciones y desarrollar planes efectivos de conservación. Los monitoreos de aves marinas muertas en las playas es un método útil para el estudio de las amenazas que afectan a las aves marinas (Harris et al. 2006). La información que se genera es útil para estimar la mortalidad por captura incidental en la actividad pesquera (Zydelis et al. 2006, Hamel et al. 2009), contaminación por petróleo (Camphuysen 1998, Camphuysen \& Heubeck 2001), interacción con basura marina (e.g., ingestión de plásticos, van Franeker et al. 2011, Acampora et al. 2014) y anomalías meteorológicas y oceanográficas (e.g., El Niño) (Schreiber 2002, Parrish et al. 2007). Estudios a largo plazo permiten determinar patrones y causas de varamientos, y desarrollar medidas de mitigación para las especies más afectadas por las actividades de pesca 
(Simons 1985, Camphuysen \& Heubeck 2001, Parrish 2005' ${ }^{1}$, Harris et al. 2006).

El Sistema Costero de Coquimbo (SCC) se ubica en el Centro Norte de Chile $\left(29^{\circ}-32^{\circ} \mathrm{S}\right)$, forma parte de la Ecorregión de la Corriente de Humboldt y por lo tanto es un área de interés global para la conservación de la biodiversidad marina (Thiel et al. 2007). Debido a la influencia del Sistema de Surgencia de la Corriente de Humboldt (SSCH) que genera una alta productividad primaria, esta zona es considerada de importancia para la conservación de aves marinas residentes y migratorias (Soazo et al. 2009). En el SCC existen siete islas costeras que son utilizadas como sitios de nidificación por varias especies de aves marinas (Luna-Jorquera et al. 2012). El ensamble de aves marinas está compuesto por $\sim 24$ especies, algunas de las cuales son endémicas del SSCH (Luna-Jorquera et al. 2003, Weichler et al. 2004). Las condiciones de productividad que favorecen a las aves marinas del SCC, también permiten el desarrollo de actividades pesqueras industriales y artesanales (Thiel et al. 2007, Montecino \& Lange 2009), lo cual genera una sobreposición espacial y temporal de las áreas de pesca y las áreas de alimentación de las aves marinas (Weichler et al. 2004, Karpouzi et al. 2007). Esta sobreposición genera una alta probabilidad de interacciones entre las artes de pesca y las aves marinas, lo que a su vez aumenta la probabilidad de muerte incidental de las aves que se manifiesta con varamientos de aves muertas en las playas (e.g., Barbraud et al. 2012). Los escasos estudios realizados en Chile han permitido cuantificar la mortalidad incidental de pingüinos y cormoranes en faenas pesqueras en el centro y sur del país (Simeone et al. 1999, Schlatter et al. 2009), junto con documentar los varamientos masivos en playas cercanas a las áreas de pesca (Brito 2002, Schlatter et al. 2009, SERNAPESCA $2013^{2}$ ), además han aportado información respecto a la ocurrencia y distribución de procelariformes (Fibla et al. 2010, Portflitt-Toro et al. 2018). Sin embargo, no existen estudios sistemáticos que permitan analizar en detalle las

${ }^{1}$ Parrish J. 2005. COASST (Coastal Observation and Seabird Survey Team) Reports 04-05: 1-27. University of Washington, Seattle.

${ }^{2}$ SERNAPESCA. 2013. Informe de inspección por denuncia ambiental: Varamiento masivo de aves marinas en Playa Los Choros, Comuna de la Higuera, Coquimbo, 5 pp. causas de la mortalidad, y determinar el efecto de la estacionalidad y comportamiento (e.g., migraciones, desplazamientos reproductivos) en las especies residentes y endémicas. Los objetivos de este estudio fueron: (a) estimar la abundancia de aves marinas muertas, (b) estimar la riqueza de especies y abundancia relativa por especie y (c) determinar el efecto estacional en la abundancia y riqueza de aves muertas en la Bahía de Coquimbo, Chile, para sentar las bases a un programa de monitoreo respecto a las causas de la mortalidad en aves marinas del Sistema Costero de Coquimbo.

\section{Materiales y Métodos}

\section{Área de ESTUdio}

Este estudio se realizó en la bahía de Coquimbo ( 295' S,), norte de Chile (Fig. 1). Esta bahía tiene una playa de arena de $\sim 20 \mathrm{~km}$ de largo y su oceanografía se caracteriza por una circulación y flujo en sentido anti horario, con una dirección de oleaje y vientos predominantes del sur oeste (Valle-Levinson et al. 2000, Valle-Levinson \& Moraga-Opazo 2006). Debido a las características de alta productividad del SCC se desarrolla una importante actividad pesquera artesanal, principalmente pesca de merluza común (Merluccius gayi gayi), jurel (Trachurus murphyi) y anchoveta (Engraulis ringens) (Thiel et al. 2007).

\section{REgistro de DATOS}

Entre mayo de 2013 y junio de 2014 se realizaron monitoreos mensuales (excepto mayo 2014) en la bahía de Coquimbo y se contabilizó el número de aves muertas por especie que se encontraban dentro de un transecto definido previamente y cuyo límite inferior se ubicó a $1 \mathrm{~m}$ sobre la línea de la marea baja y el límite superior a 7 ó $10 \mathrm{~m}$ sobre la línea de la marea alta. El estado de descomposición de los cadáveres de aves se clasificó en 4 categorías: (1) Fresco: la data de muerte es reciente $(<24 \mathrm{~h})$, con nula o baja intervención de carroñeros, con presencia intacta de los órganos; (2) Descomposición moderada: la data de muerte no es mayor a 2 o 3 días, aun presenta plumas firmes, tejido relativamente fresco, sin los órganos viscerales o parcialmente destrozados; (3) Descomposición avanzada: la data de muerte es superior a una semana, las plumas se sueltan al manipular el ave y el tejido está descompuesto, sin órganos, presencia de larvas de moscas y coleópteros del género Phaleria; y (4) Huesos: el ave sólo presenta el esqueleto, sin plumas ni tejido. En cada ocasión se examinó el cadáver para determinar la 
presencia de lesiones tales como: cortes en las patas o alas ocasionadas por redes de pesca (Schlatter et al. 2009) y presencia de hidrocarburos en las plumas. Para evitar el doble conteo de un mismo ejemplar en monitoreos posteriores, los cadáveres fueron enterrados a $\sim 5 \mathrm{~m}$ de distancia por sobre el transecto.

\section{ANÁlisis de DATOS}

Se calculó la riqueza $(\mathrm{S})$ de especies como el número de especies identificadas para cada mes y cada estación climática del año, durante el periodo de estudio. Los cadáveres que no lograron ser identificados no fueron utilizados en el análisis. La abundancia se expresó de dos maneras: (1) el número total y por especie de los cadáveres encontrados en cada mes y estación del año, y (2), para comparar los resultados con información de la literatura, se calculó la media aritmética de la densidad total de aves $\mathrm{km}^{-1}$ sobre la base de los datos mensuales y la extensión del transecto utilizado (162,6 km). Para examinar el efecto de la estacionalidad sobre la mortalidad, los datos del número de aves, total y por especie, se agruparon en estaciones: otoño (marzo-abril-mayo), invierno (juniojulio-agosto), primavera (septiembre-octubre-noviembre) y verano (diciembre-enero-febrero). Todos los análisis se hicieron en el programa libre R Studio (R Core Team 2017). La mortalidad total por temporada, independientemente de la especie, se analizó mediante modelos lineales generalizados (GLM) con una distribución binomial negativa para controlar la sobre dispersión de las varianzas (Zuur et al. 2009). El GLM se hizo utilizando el paquete MASS (Ripley et al. 2013). Para analizar el efecto de la estacionalidad en la composición de especies de aves marinas varadas se aplicó un GLM multivariado utilizando la función manyglm disponible en el paquete mvabund (Wang et al. 2012). La validación del modelo se realizó mediante un análisis de la devianza utilizando el método de re-muestreo 'PIT-trap' (probability integral transform residual bootstrap) con 9999 iteraciones. La inspección visual de los residuales no reveló ninguna desviación evidente de homocedasticidad o normalidad. La Figura 2 se realizó usando el paquete ggplot2 (Wickham 2016).

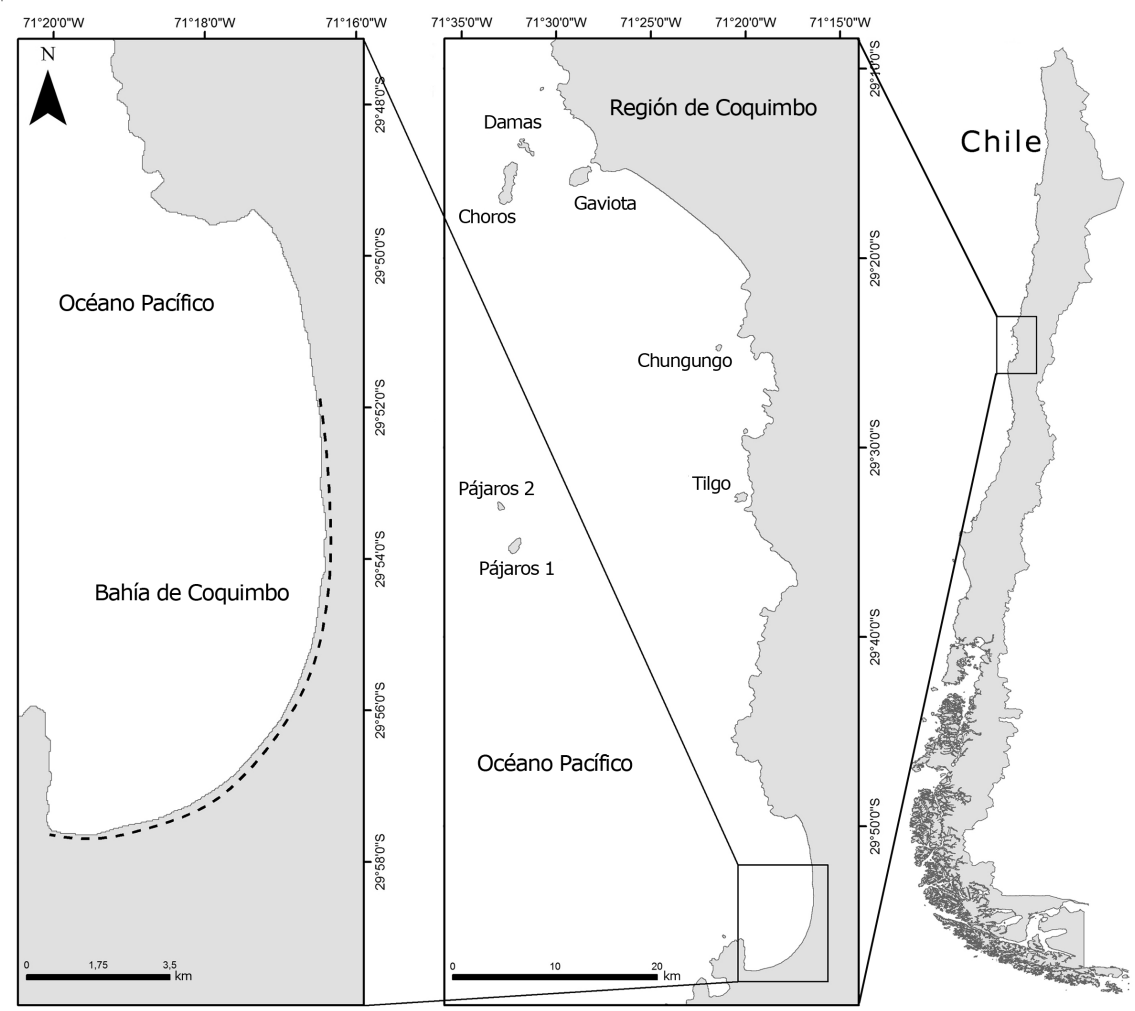

Figura 1. Sistema de islas costeras de la Región de Coquimbo (recuadro derecho) y sitio de estudio en la bahía de Coquimbo (línea segmentada, recuadro izquierdo) / System of coastal islands of the Coquimbo Region (right box) and study site in Coquimbo Bay (segmented line, left box) 


\section{Resultados}

Se determinaron 395 ejemplares de aves marinas muertas, de las cuales 13 no fueron identificadas al nivel de especie debido a su avanzado estado de descomposición. La media de la densidad (con una desviación estándar) de aves varadas fue de 2,3 $\pm 1,6$ aves $\mathrm{km}^{-1}(95 \% \mathrm{IC}=1,82$ 2,71). Los 382 ejemplares identificados correspondieron a 21 especies que se agruparon en los siguientes ordenes: $56,5 \%(n=216)$ pertenecían al orden Suliformes (i.e., cormoranes y piqueros $), 20,7 \%(n=79)$ al orden Charadriiformes (i.e., gaviotas, gaviotines), 8,9\% $(\mathrm{n}=34)$ al orden Pelecaniformes (i.e., pelícanos), 8,4\% $(n=32)$ al orden Sphenisciformes (i.e., pingüinos), y 5,5\% $(\mathrm{n}=21)$ al orden Procellariiformes (i.e., fardelas, petreles y albatros)
Las especies más abundantes fueron el piquero (Sula variegata, 30\% del total de cadáveres identificados), el cormorán guanay (Phalacrocorax bougainvillii, 21\%), la gaviota dominicana (Larus dominicanus, 17\%), el pelícano (Pelecanus thagus, 9\%) y el pingüino de Humboldt (Spheniscus humboldti, 8\%) (Tabla 1). De estas 5 especies sólo el pelícano no nidifica en las islas del SCC. El piquero apareció en las playas durante todo el período de estudio a diferencia de otras especies como el petrel paloma de pico ancho, el petrel paloma de pico delgado, la fardela blanca o el petrel moteado que fueron registradas sólo una vez.

Tabla 1. Abundancia de aves marinas muertas encontradas en la bahía de Coquimbo / Abundance of dead's seabirds and species found in Coquimbo Bay

\begin{tabular}{|c|c|c|c|}
\hline Orden & Especie (categoría UICN) & Nombre común & $\begin{array}{l}\text { Abundancia } \\
\text { total }\end{array}$ \\
\hline \multirow[t]{4}{*}{ Suliformes } & Sula variegata $(\mathrm{LC})$ & Piquero & 115 \\
\hline & $\begin{array}{l}\text { Phalacrocorax bougainvillii } \\
\text { (NT) }\end{array}$ & Cormorán guanay & 83 \\
\hline & Phalacrocorax gaimardi (NT) & Cormorán lile & 10 \\
\hline & Phalacrocorax brasilianus (LC) & Cormorán yeco & 8 \\
\hline \multirow[t]{5}{*}{ Charadriiformes } & Larus dominicanus (LC) & Gaviota dominicana & 65 \\
\hline & Leucophaeus modestus (LC) & Gaviota garuma & 5 \\
\hline & Larosterna inca $(\mathrm{NT})$ & Gaviotín monja & 5 \\
\hline & Sterna hirundinacea $(\mathrm{LC})$ & Gaviotín sudamericano & 1 \\
\hline & Haematopus ater (LC) & Pilpilén negro & 3 \\
\hline Pelecaniformes & Pelecanus thagus (NT) & Pelícano & 34 \\
\hline \multirow[t]{2}{*}{ Sphenisciformes } & Spheniscus humboldti (VU) & Pingüino de Humboldt & 31 \\
\hline & Spheniscus magellanicus (NT) & Pingüino de Magallanes & 1 \\
\hline \multirow[t]{9}{*}{ Procellariiformes } & Ardenna grisea $(\mathrm{NT})$ & Fardela negra & 7 \\
\hline & Macronectes halli (LC) & Petrel gigante subantártico & 4 \\
\hline & Pelecanoides garnotii (EN) & Yunco & 4 \\
\hline & Daption capense (LC) & Petrel moteado & 1 \\
\hline & Macronectes giganteus $(\mathrm{LC})$ & Petrel gigante antártico & 1 \\
\hline & Pachyptila belcheri (LC) & Petrel paloma de pico delgado & 1 \\
\hline & Pachyptila vittata (LC) & Petrel paloma de pico ancho & 1 \\
\hline & Ardenna creatopus (VU) & Fardela blanca & 1 \\
\hline & Thalassarche melanophris (NT) & Albatros de ceja negra & 1 \\
\hline Total & & & 382 \\
\hline
\end{tabular}

UICN: Categoría de conservación según la lista roja de la Unión Internacional para la Conservación de la Naturaleza. EN: En peligro; VU: Vulnerable; NT: Casi amenazado; LC: Preocupación menor 
No se determinó efecto de la estacionalidad sobre el número de aves muertas en las playas (devianza $=80,56$; $P=0,155$; grados de libertad= 3 y 9). En términos generales, los piqueros fueron más abundantes en primavera $(n=36)$ y verano $(n=35)$ y la gaviota dominicana fue más abundante en verano $(n=20)$, pero no se determinó un efecto significativo de la estacionalidad sobre la riqueza de especies (test multivariado de Wald $=6,177 ; P$ $=0,195)$. Sin embargo, al resumir cada término multivariado para la estacionalidad ajustada por el GLM, se determinó una disminución significativa en la cantidad de cadáveres de cormorán guanay y cormorán lile en verano (test de Wald=4,459; $P=0,036$; Fig. 2).

En 17 ejemplares $(\sim 4,5 \%$ del total $)$, principalmente cormorán guanay $(n=16)$ y un ejemplar de cormorán lile, fue identificada la causa de muerte como atribuible a interacción con actividades pesqueras. Los cormoranes guanay estaban en estado 1 (ver arriba), no presentaban marcas atribuibles al enredo en redes de pesca o presencia de hidrocarburos. Al analizar el contenido estomacal la totalidad presentó el estómago lleno con anchovetas enteras y algunas semi-digeridas, también peces en el esófago y en el pico, lo cual sugiere muerte por ahogamiento mientras se alimentaban. El ejemplar de cormorán lile tenía una red de pesca enredada en el tarso derecho el cual había cercenado el hueso, el estómago no estaba presente. Además, se examinó un petrel paloma de pico delgado y un petrel paloma de pico ancho, los cuales tenían fragmentos de plásticos en sus estómagos y no presentaban restos de comida en el intestino ni el estómago.
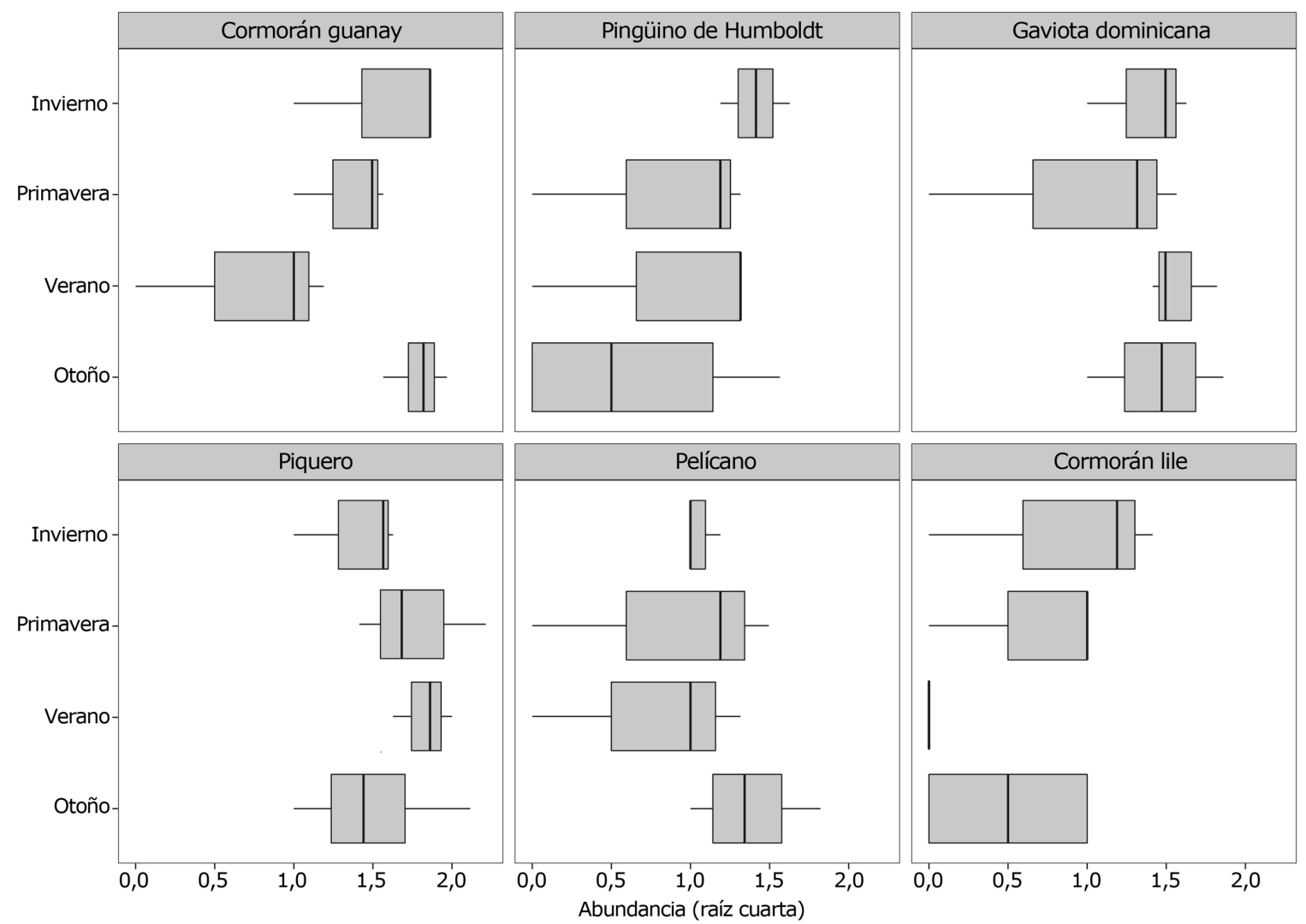

Figura 2. Boxplot de la abundancia (raíz cuarta) estacional de las especies con mayor mortalidad en las playas de la bahía de Coquimbo, Chile / Boxplot of the abundance (fourth root) seasonal of the species with greater mortality in the beaches of Coquimbo Bay, Chile 


\section{Discusión}

El monitoreo de aves varadas en las playas es útil para cuantificar tanto mortalidad aguda (e.g., derrames de petróleo) como crónica (e.g., mortalidad incidental en artes de pesca) (Ford 2006) a distintas escalas espaciales (Parrish et al. 2007, Zydelis et al. 2013). Sin embargo, la deriva de los cadáveres hacia la playa es influida por las condiciones oceanográficas, en especial por la dirección e intensidad del viento (Bibby \& Lloyd, 1977, Hlady \& Burger 1993, Flint \& Fowler 1998, Wiese 2003). El tamaño corporal, la acción de depredadores (Hunter 1983) y el tiempo a la deriva en el mar son factores que influyen en la boyantes y por tanto la probabilidad que los cadáveres lleguen a las playas. Estudios experimentales sugieren que el número de aves muertas que se encuentra en las playas representa un 11 a $58 \%$ del total que muere en el mar (Bibby \& Lloyd 1977), y puede llegar incluso a un $10 \%$ en el caso de animales que mueren lejos (35 a $100 \mathrm{~km})$ de la costa (Hlady \& Burger 1993). Adicionalmente, al llegar a la playa los cadáveres pueden ser consumidos por carroñeros lo que dificulta la identificación de la especie y la determinación de las causas de la muerte (Stephen \& Burger 1994), como ocurrió en este estudio en que la acción de carroñeros (jotes y perros) dificultó la identificación de un 3,3\% del total de aves varadas. Todos estos factores se deben tener en cuenta en la estimación final de la mortalidad en un programa de monitoreo de largo alcance.

En este estudio se encontraron 395 aves muertas agrupadas en 21 especies. Esta riqueza de especies es similar a las 24 especies reportadas para el SCC (Weichler et al. 2004), pero es alta en comparación a lo reportado en estudios similares realizados en Chile. En la Región de Antofagasta se encontraron 241 aves muertas agrupadas en 8 especies (Fibla et al. 2012) y en la Región de Valparaíso el número de aves muertas fue de $\sim 692$ agrupadas en 4 especies, de las cuales el $95 \%$ correspondió a pingüinos (Simeone et al. 1999). Se sugiere que la cercanía y tamaño de las colonias es un factor que influye en el número y abundancia de las especies de aves muertas en las playas de Coquimbo (Van Pelt \& Piatt 1995, Harris et al. 2006) y probablemente la riqueza de especies. Cercanas al área de estudio hay islas (ver Simeone et al. 2003, Luna-Jorquera et al. 2012) en las cuales nidifican 7 especies (pingüino de Humboldt, piquero, yunco, guanay, lile, yeco y gaviota dominicana) de 21 especies que vararon en las playas de Coquimbo, lo cual también explicaría que el 82,5\% del total de aves muertas corresponden a estas 7 especies. Otro factor que explicaría la riqueza de especies observadas, se relaciona con la productividad de las aguas del SCC que atrae a especies que nidifican en otras latitudes (Weichler et al. 2004, Thiel et al. 2007), tales como el albatros de ceja negra, la fardela blanca, el petrel paloma de pico ancho, el petrel paloma de pico delgado y el petrel moteado.

La densidad media de cadáveres en las playas en este estudio $\left(2,3\right.$ aves $\left.\cdot \mathrm{km}^{-1}\right)$ es menor que la reportada para la costa del Pacífico norte en Estados Unidos estimada en $8,5 \pm 1,4$ aves $\cdot \mathrm{km}^{-1}$ para un periodo de más de 4 años (Parrish 2005) $)^{1}$, pero es más alto que las 0,22 $\pm 0,04$ aves $\mathrm{km}^{-1}$ encontradas en la costa noroeste del océano Atlántico (Massachusetts) para un periodo de 2 años (Harris et al. 2006). Los resultados obtenidos se comparan mejor con lo reportado para Bélgica de 2,6 $\pm 2,1$ aves $\cdot \mathrm{km}^{-1}$ para un periodo de 6 años (1993 a 1999) (Seys et al. 2002) y para Holanda de 2 a 18 aves $\cdot \mathrm{km}^{-1}$ (Camphuysen \& Heubeck 2001). En ambos estudios, la causa de muerte de las aves estuvo asociada principalmente a empetrolamiento.

En este estudio, la causa de muerte parece estar relacionada con la interacción de las actividades pesqueras. Así lo sugieren las observaciones hechas en los cormoranes en los cuales se determinó con certeza la causa de muerte. Además, un porcentaje alto $(\sim 82 \%)$ de las aves muertas correspondieron a especies buceadoras (i.e., pingüinos, cormoranes, yuncos, piqueros), altamente vulnerables a morir ahogadas en redes de pesca, sobre todo de enmalle (Zydelis et al. 2013). En la Región de Coquimbo algunas de las principales presas objetivos de la pesca artesanal serían la anchoveta y la merluza, capturadas con redes de cerco y enmalle, en las cuales cuando se alimentan de estos recursos quedan atrapadas principalmente aves buceadoras, tales como piqueros, guanayes, pelícanos (Luna-Jorquera et al. 2015) ${ }^{3} \mathrm{y}$ pingüinos (Simeone et al. 1999). En 2013 se registró la muerte de $\sim 610$ ejemplares de cormorán guanay mientras se alimentaban de anchovetas, sin embargo, no se pudo determinar una causa de muerte, aunque se le atribuye una causa común a la mayoría de las aves encontradas muertas (SERNAPESCA 2013) ${ }^{2}$. Una de las posibles causas es la pesca con explosivos, que en otras partes del mundo causa grandes daños al ecosistema marino

\footnotetext{
${ }^{3}$ Luna-Jorquera G, J Arata, O Yates, C Suazo, M Sepúlveda, D Miranda-Urbina, E Acuña \& S Zúñiga. 2015. Caracterización de los efectos de las principales pesquerías artesanales en los ecosistemas marinos. Licitación 2013-115-dap-35, Subsecretaría de Pesca, Gobierno de Chile, 236 pp.
} 
(Guard \& Masaiganah 1997). Otra causa de mortalidad de aves marinas se relaciona con derrames de petróleo (Camphuysen 1998), pero en este estudio no se encontró evidencia de empetrolamiento. Además, la presencia de perros en las playas parece ser la causa de muerte de gaviotas dominicanas, las cuales son atacadas mientras descansan en las playas (Chávez-Villavicencio et al. 2015, Aguilar et al. 2016).

La abundancia de algunas especies de aves marinas (e.g., piquero) en el SCC aumenta durante los periodos reproductivos (e.g., primavera-verano) (Weichler et al. 2004) y en otras especies más comunes de observar, como la gaviota dominicana, se mantiene durante el año (Yorio et al. 2016). El número de aves muertas en las playas parece ser relativamente constante a lo largo del año ya que no se encontró un efecto significativo de la estacionalidad. Al parecer el piquero es la especie más afectada en primavera y verano, que es la época del año en la cual se reproducen y se alimentan en grandes grupos cerca de la costa (Simeone et al. 2003, Weichler et al. 2004, Ludynia et al. 2005). En otras regiones de Chile se ha registrado mortalidad asociada a factores estacionales, tal es el caso de 1.380 pingüinos de Magallanes que murieron en redes de enmalle en las costas de Queule, Chiloé, durante su viaje de invierno hacia el norte (Schlatter et al. 2009), también se suma el caso de la mortalidad masiva ( 1000-2500 animales muertos) de fardela negra (Ardenna griseus) en 2001, 2002 y 2012 registradas en la Región de Valparaíso, durante el periodo de migración (otoño-invierno) hacia el norte (Brito 2002, $2012^{4}$ ). En las dos especies la muerte de las aves fue atribuida a la interacción con pesquería de cerco.

Se sugiere que en el SCC la mortalidad por interacción con las actividades pesqueras es crónica y subestimada (ver arriba) y afecta principalmente a las especies buceadoras que nidifican en las islas. Además, la

\footnotetext{
${ }^{4}$ Brito JL. 2012. Informe sobre la mortalidad de aves marinas en la costa de Cartagena y Santo Domingo en la provincia de San Antonio. Museo Municipal de Ciencias Naturales y Arqueología de San Antonio, 9 pp.

${ }^{5}$ Dec. Ex. N ${ }^{\circ}$ 564-2017 Establece Veda Biológica para el Recurso Anchoveta, III-IV Región y Fija Porcentaje de Especies como Fauna Acompañante que Señala. (F.D.O. 25-08-2017) <http:// www.subpesca.cl/portal/615/w3-article-98057.html>
}

mortalidad incidental de aves marinas se debe principalmente a la interacción con las actividades pesqueras, especialmente en la pesquería de la anchoveta que opera durante la mayor parte del año excepto durante los periodos de veda generalmente entre agosto y septiembre (Decreto Exento N564-2017 SUBPESCA) ${ }^{5}$. Considerando la intensa actividad pesquera artesanal que se desarrolla en la Región de Coquimbo, un programa de monitoreo permitiría cuantificar y correlacionar, a un costo relativamente bajo, el número de aves muertas con los diferentes factores que determinan la mortalidad, particularmente de las especies que además enfrentan otras amenazas en sus colonias de nidificación (i.e., especies exóticas invasivas, extracción de guano) (Simeone et al. 2003, Luna-Jorquera et al. 2012). Finalmente, los resultados sugieren que la mortalidad de aves marinas en el SCC es crónica y que podría estar relacionada con la captura incidental en actividades pesquera, por lo tanto es importante desarrollar e implementar medidas que reduzcan la mortalidad incidental de las aves marinas endémicas del SSCH con problemas de conservación.

\section{Agradecimientos}

Agradecemos el apoyo brindado por la Sala de Colecciones Biológicas de la Universidad Católica del Norte (SCBUCN), Coquimbo, Chile. Este estudio forma parte de la Red de Varamientos de Aves Marinas - REVAM y se realizó como parte de la tesis de MP-T para optar al título de Biólogo Marino. Agradecemos también el aporte de los evaluadores anónimos por sus comentarios y sugerencias al escrito.

\section{LITERATURA CITADA}

Acampora H, QA Schuyler, KA Townsend \& BD Hardesty. 2014. Comparing plastic ingestion in juvenile and adult stranded short-tailed shearwaters (Puffinus tenuirostris) in eastern Australia. Marine Pollution Bulletin 78: 63-68.

Aguilar R, A Simeone, J Rottmann, M Perucci \& G LunaJorquera. 2016. Unusual coastal breeding in the desertnesting Gray Gull (Leucophaeus modestus) in Northern Chile. Waterbirds 39: 69-73.

Barbraud C, V Rolland, S Jenouvrier, M Nevoux, K Delord \& H Weimerskirch. 2012. Effects of climate change and fisheries bycatch on Southern Ocean seabirds: a review. Marine Ecology Progress Series 454: 285-307.

Bibby CJ \& CS Lloyd. 1977. Experiments to determine the fate of dead birds at sea. Biological Conservation 12: 295309. 
Brito JL. 2002. Muertes de Puffinus griseus en redes de pesca de cerco industrial y artesanal en la costa de San Antonio, Región de Valparaíso, Chile. Boletín Chileno de Ornitología 9: 33-34.

Camphuysen KCJ. 1998. Beached bird surveys indicate decline in chronic oil pollution in the North Sea. Marine Pollution Bulletin 36: 519-526.

Camphuysen KCJ \& M Heubeck. 2001. Marine oil pollution and beached bird surveys: the development of a sensitive monitoring instrument. Environmental Pollution 112: 443461.

Chávez-Villavicencio $\mathrm{C}, \mathrm{P}$ Molina-Pérez \& E TabiloValdivieso. 2015. Respuesta de la riqueza de aves en presencia de visitantes, vehículos y perros en el humedal 'El Culebrón', Chile. The Biologist, Lima 13: 313-327.

Croxall JP, SHM Butchart, B Lascelles, AJ Stattersfield, B Sullivan, A Symes \& P Taylor. 2012. Seabird conservation status, threats and priority actions: a global assessment. Bird Conservation International 22: 1-34.

Fibla P, N Sallaberry-Pincheira \& M Sallaberry. 2010. Conteo de aves marinas muertas en la playa La Rinconada, Antofagasta y comentario acerca de la distribución de Macronectes halli en Chile. Boletín Chileno de Ornitología 16: 37-41.

Guard M \& M Masaiganah. 1997. Dynamite fishing in Southern Tanzania, geographical variation, intensity of use and possible solutions. Marine Pollution Bulletin 34: 758762.

Hamel N, A Burger, K Charleton, P Davidson, S Lee, DF Bertram \& JK Parrish. 2009. Bycatch and beached birds: assessing mortality impacts in coastal net fisheries using marine bird strandings. Marine Ornithology 37: 41-60.

Harris RJ, FS Tseng, MA Pokras, BA Suedmeyer, JSH Bogart, RL Prescott \& SH Newman. 2006. Beached bird surveys in Massachusetts: the Seabird Ecological Assessment Network (SEANET). Marine Ornithology 122: 115-122.

Karpouzi VS, R Watson \& D Pauly. 2007. Modelling and mapping resource overlap between seabirds and fisheries on a global scale: a preliminary assessment. Marine Ecology Progress Series 343: 87-99.

Ludynia K, S Garthe \& G Luna-Jorquera. 2005. Seasonal and regional variation in the diet of the kelp gull Larus dominicanus in northern Chile. Waterbirds 28: 359-365.

Luna-Jorquera G, A Simeone \& R Aguilar. 2003. Ecofisiología de animales endotermos en un desierto cálido y un mar frío: El caso de las aves marinas de la corriente de Humboldt. En: Bozinovic F (ed). Fisiología ecológica y evolutiva. Teoría y casos de estudios en animales, pp. 297316. Ediciones Universidad Católica de Chile, Santiago.

Luna-Jorquera G, CE Fernández \& MM Rivadeneira. 2012. Determinants of the diversity of plants, birds and mammals of coastal islands of the Humboldt Current systems: implications for conservation. Biodiversity and Conservation 21: 13-32.

Montecino V \& CB Lange. 2009. The Humboldt Current System: Ecosystem components and processes, fisheries, and sediment studies. Progress in Oceanography 83: 6579.

Parrish J, N Bond, H Nevins, N Mantua, R Loeffel, W Peterson \& J Harvey. 2007. Beached birds and physical forcing in the California Current System. Marine Ecology Progress Series 352: 275-288.

Portflitt-Toro M, D Miranda-Urbina \& G Luna-Jorquera. 2018. Specimen record confirms Broad-billed Prion Pachyptila vittata presence in Chilean waters. Marine Ornithology 46: 69-70.

R Core Team. 2017. R: A language and environment for statistical computing. R Foundation for Statistical Computing, Vienna. <https://www.R-project.org/>

Ripley B, B Venables, DM Bates, K Hornik, A Gebhardt, D Firth \& MB Ripley. 2013. MASS: Support functions and datasets for Venables and Ripley's MASS. Package MASS. $<$ http://cran. r-project.org/web/packages/MASS/ index.html $>$

Schlatter R, E Paredes, J Ulloa, J Harris, A Romero, J Vasquez, A Lizama, C Hernández \& A Simeone. 2009. Mortandad de Pingüino de Magallanes (Spheniscus magellanicus) en Queule, Región de La Araucanía, Chile. Boletín Chileno de Ornitología 15: 78-86.

Schreiber EA. 2002. Climate and weather effects on seabirds. En: Schreiber EA \& J Burger (eds). Biology of marine birds, pp. 179-207. CRC Press, Boca Raton.

Seys J, H Offringa, J Van Waeyenberge, $P$ Meire \& E Kuijken. 2002. An evaluation of beached bird monitoring approaches. Marine Pollution Bulletin 44: 322-33.

Simeone A, M Bernal \& J Meza. 1999. Incidental mortality of Humboldt Penguins Spheniscus humboldti in gill nets, central Chile. Marine Ornithology 27: 157-161.

Simeone A, G Luna-Jorquera, M Bernal, S Garthe, F Sepúlveda, R Villablanca, U Ellenberg, M Contreras, J Muñoz \& T Ponce. 2003. Breeding distribution and abundance of seabirds on islands off north- central Chile. Revista Chilena de Historia Natural 76: 323-333.

Simons MM. 1985. Beached bird survey project on the Atlantic and Gulf coasts. American Birds 39: 358-362.

Soazo PO, I Rodríguez-Jorquera, P Arrey-Garrido \& A Jaramillo. 2009. Chile. In: Devenish C, DF Díaz Fernández, RP Clay, I Davidson \& I Yépez-Zabala (eds). Important bird areas of the Americas. Priority sites for biodiversity conservation, BirdLife Conservation Series 16: 125-134. BirdLife International, Quito.

Stephen C \& AE Burger. 1994. A comparison of two methods for surveying mortality of beached birds in British Columbia. The Canadian Veterinary Journal 35: 631-635. 
Thiel M, E Macaya, E Acuña, W Arntz, H Bastias, K Brokordt, P Camus, JC Castilla, L Castro, M Cortés, CP Dumont, R Escribano, M Fernandez, J Gajardo, C Gaymer, I Gomez, A González, H González, P Haye, J Illanes, J Iriarte, D Lancellotti, G Luna-Jorquera, C Luxoro, P Manriquez, V Marín, P Muñoz, S Navarrete, E Perez, E Poulin, J Sellanes, H Sepúlveda, W Stotz, F Tala, A Thomas, C Vargas, J Vásquez \& A Vega. 2007. The Humboldt Current system of northern and central Chile oceanographic processes, ecological interactions and socioeconomic feedback. Oceanography and Marine Biology: An Annual Review 45: 195-344.

Valle-Levinson A, J Moraga, J Olivares \& JL Blanco. 2000. Tidal and residual circulation in a semi-arid bay: Coquimbo Bay. Chile. Continental Shelf Research 20: 2009-2028.

Valle-Levinson A \& J Moraga-Opazo. 2006. Observations of bipolar residual circulation in two equatorward-facing semiarid bays. Continental Shelf Research 26: 179-193.

Van Franeker J, C Blaize, J Danielsen, K Fairclough, J Gollan, N Guse, PL Hansen, M Heubeck, JK Jensen, G Le Guillou, B Olsen, KO Olsen, J Pedersen, EWM Stienen \& DM Turner. 2011. Monitoring plastic ingestion by the northern fulmar Fulmarus glacialis in the North Sea. Environmental Pollution 159: 2609-2615.

Van Pelt TI \& JF Piatt. 1995. Deposition and persistence of beachcast seabird carcasses. Marine Pollution Bulletin 30: 794-802.
Wang Y, U Naumann, ST Wright \& DI Warton. 2012. mvabund- an $\mathrm{R}$ package for model-based analysis of multivariate abundance data. Methods in Ecology and Evolution 3: 471-474.

Weichler T, S Garthe, G Luna-Jorquera \& J Moraga. 2004. Seabird distribution on the Humboldt Current in northern Chile in relation to hydrography, productivity, and fisheries. ICES Journal of Marine Science 61: 148-154.

Wickham H. 2016. ggplot2. Elegant graphics for data analysis, 213 pp. Springer-Verlag, New York.

Yorio P, JO Branco, J Lenzi, G Luna-Jorquera \& C Zavalaga. 2016. Distribution and trends in kelp gull (Larus dominicanus) coastal breeding populations in South America. Waterbirds 39: 114-135.

Zuur AF, EN Ieno, NJ Walker, AA Saveliev \& GM Smith. 2009. Zero-truncated and zero-inflated models for count data. In Mixed effects models and extensions in ecology with R, pp. 261-293. Springer New York.

Zydelis R, M Dagys \& G Vaitkus. 2006. Beached bird surveys in Lithuania reflect marine oil pollution and bird mortality in fishing nets. Marine Ornithology 34: 161-166.

Zydelis R, C Small \& G French. 2013. The incidental catch of seabirds in gillnet fisheries: A global review. Biological Conservation 162: 76-88.

Editor: Claudia Bustos D. 